\title{
Fate of pulmonary nodules detected by computer-aided diagnosis and physician review on the computed tomography simulation images for hepatocellular carcinoma
}

\author{
Hyojung Park, MD, Jin-Sung Kim, PhD, Hee Chul Park, MD, PhD, Dongryul Oh, MD \\ Department of Radiation Oncology, Samsung Medical Center, Sungkyunkwan University School of Medicine, Seoul, Korea
}

\begin{abstract}
Purpose: To investigate the frequency and clinical significance of detected incidental lung nodules found on computed tomography (CT) simulation images for hepatocellular carcinoma (HCC) using computer-aided diagnosis (CAD) and a physician review.

Materials and Methods: Sixty-seven treatment-naïve HCC patients treated with transcatheter arterial chemoembolization and radiotherapy (RT) were included for the study. Portal phase of simulation CT images was used for CAD analysis and a physician review for lung nodule detection. For automated nodule detection, a commercially available CAD system was used. To assess the performance of lung nodule detection for lung metastasis, the sensitivity, negative predictive value (NPV), and positive predictive value (PPV) were calculated.

Results: Forty-six patients had incidental nodules detected by CAD with a total of 109 nodules. Only 20 (18.3\%) nodules were considered to be significant nodules by a physician review. The number of significant nodules detected by both of CAD or a physician review was 24 in 9 patients. Lung metastases developed in 11 of 46 patients who had any type of nodule. The sensitivities were $58.3 \%$ and $100 \%$ based on patient number and on the number of nodules, respectively. The NPVs were $91.4 \%$ and $100 \%$, respectively. And the PPVs were $77.8 \%$ and $91.7 \%$, respectively.

Conclusion: Incidental detection of metastatic nodules was not an uncommon event. From our study, CAD could be applied to CT simulation images allowing for an increase in detection of metastatic nodules.
\end{abstract}

Keywords: Computer-assisted diagnosis, Radiotherapy, Hepatocellular carcinoma

\section{Introduction}

Computed tomography (CT)-based treatment planning before radiation therapy (RT) has become standard practice. CT simulation and three-dimensional treatment planning for RT offer more accurate targeting, improved dose homogeneity and avoidance of critical organs. Some investigators have also reported that the CT simulation images used in RT could be used to detect unexpected abnormalities [1-4].

In general, incidental findings detected on CT simulation images are rare [3]. However, lung metastases from hepatocellular carcinoma (HCC) are not infrequent and are

Received 18 April 2014, Revised 4 June 2014, Accepted 25 June 2014.

Correspondence: Hee Chul Park, MD, PhD, Department of Radiation Oncology, Samsung Medical Center, Sungkyunkwan University School of Medicine, 81 Irwon-ro, Gangnam-gu, Seoul 135-710, Korea. Tel: +82-2-3410-2605, Fax: +82-2-3410-2619, E-mail: rophc@skku.edu

(c) This is an Open Access article distributed under the terms of the Creative Commons Attribution Non-Commercial License (http://creativecommons.org/ licenses/by-nc/3.0/) which permits unrestricted non-commercial use, distribution, and reproduction in any medium, provided the original work is properly cited.

www.e-roj.org 
sometimes detected on CT simulation images before their definitive diagnosis. Therefore, it is necessary for radiation oncologists to review CT simulation images carefully.

Image review was performed by a diagnostic radiologist in most of the previous studies. However, in a real clinical situation, CT simulation images are not routinely interpreted by a radiologist because of heavy workloads. With advances in digital imaging technologies, approaches to automated computer diagnosis to aid in the decision making process of the radiologist have emerged. Computer-aided diagnosis (CAD) was developed for computerized analysis of medical images in the 1960s [5]. CAD systems rely on a discrimination mechanism based on densitometric gray-level thresholding and recognize nodules as opaque lesions surrounded by lung parenchymal attenuation [6]. In diagnostic radiology, CAD is used to improve the diagnostic accuracy of medical images and the performance of the radiologist.

The purpose of this study was to investigate the clinical significance of undetected incidental lung nodules found on CT simulation images assisted by CAD in HCC patients, thereby assessing the feasibility of using CAD to review CT simulation images in the clinical setting of a radiation oncology practice.

\section{Materials and Methods}

\section{Eligibility}

Treatment-naïve HCC patients treated with scheduled interval transcatheter arterial chemoembolization (TACE) and RT between March 2008 and December 2010 were reviewed retrospectively. The eligibility criteria included unresectable $\mathrm{HCC}$, clinical liver function with a Child-Pugh score of $A$ or $B$, and no previous history of treatment for any other malignancy. Patients who had an Eastern Cooperative Oncology Group performance status of more than 2 or an extrahepatic metastasis at diagnosis were excluded.

\section{Treatment and follow-up protocol}

All patients were initially treated by TACE. On day 8 after TACE, all patients underwent pre-RT clinical examinations which included a physical examination and biochemical profiles including a liver function test. If the results of the pre-RT clinical examinations indicated that the patient was fit to undergo RT, CT simulation was performed on the same day. RT was initiated on day 14 after TACE. Regular follow-up liver CTs were done after RT. The first follow-up liver CT was performed after 1 month and thereafter at 3- to 4-month intervals.

\section{Computed tomography simulation and computer-aided diagnosis}

CT simulation was performed with a LightSpeed RT16 (GE Healthcare, Milwaukee, WI, USA) helical scanner set at 120 $\mathrm{kVp}$, auto $\mathrm{mA}$ and 5-mm slice thickness. Intravenous contrast media (Visipaque 270, Amersham Health Inc., UK; $2 \mathrm{~mL} / \mathrm{kg}$ to a maximum of $200 \mathrm{~mL}$ ) was delivered at a rate of $5 \mathrm{~mL} / \mathrm{sec}$, and exhale breath-hold CT scans were obtained at 25 to 30 seconds intervals (arterial phase) and 50 to 60 seconds (portal phase) after the injection. Following this, 4-dimensional CT scans were acquired by a retrospective binning method using a Real-time Position Management (RPM) system (Varian Medical System, Palo Alto, CA, USA). The CT simulation utilized a scan range from $5 \mathrm{~cm}$ above the diaphragm to $5 \mathrm{~cm}$ below the liver. The portal phase image data in Digital Imaging and Communications in Medicine (DICOM) format were digitally transferred from the CT scanner to a personal computer for image analysis. For automated lung nodule detection, a commercially available CAD system (Xelis-Lung Solution; INFINITT Healthcare Corp., Phillipsburg, NJ, USA) was used. This provided automatic 3-dimensional nodule volume measurements in the lung region and other key statistics useful in nodule classification (Fig. 1).

\section{Review of the computed tomography simulation images} All CT simulation images were analyzed using CAD for automated nodule detection. Following this analysis, a radiation oncologist reviewed the CT simulation images without the assistance of CAD. And the nodules detected by CAD were categorized by a physician. The final results of CT simulation image review were assembled through the integration of the results from the image reviews performed using CAD and by a physician.

The diagnosis of a lung metastasis was based on the increment of nodule size and the morphological characteristics of the nodule. Typical radiologic findings of a lung metastasis include multiple peripherally located round variable-sized nodules and diffuse thickening of the interstitium $[7,8]$. To focus on the detection of synchronous lung metastases, only CT images obtained within 4 months after RT completion were used for diagnosis of lung metastasis.

\section{Statistical analysis}

To assess the performance of CAD and a physician in the detection of metastatic lung nodules, the sensitivity, false positive (FP) fraction, negative predictive value (NPV) and 
A

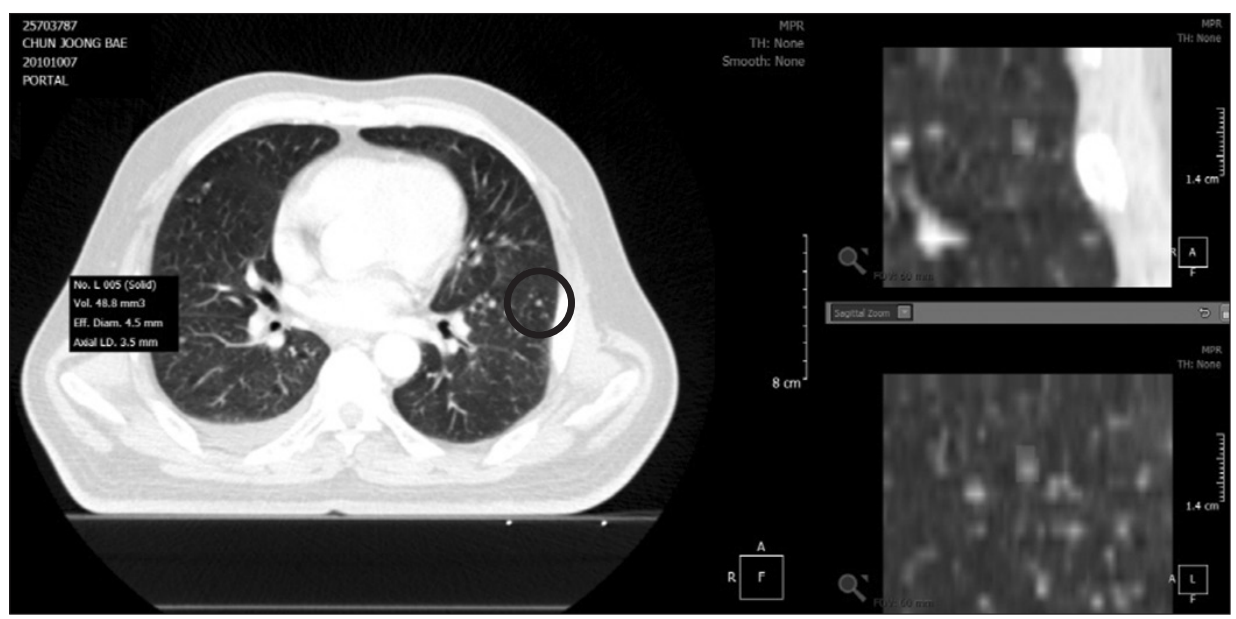

B

\begin{tabular}{rrccc}
$\#$ & Type & Volume $\left(\mathrm{mm}^{3}\right)$ & Eff.Diam. $(\mathrm{mm})$ & Axial LD. $(\mathrm{mm})$ \\
\hline$\triangle L 005$ & Solid & 48.8 & 4.5 & 3.5 \\
$\triangle \mathrm{R} 004$ & Solid & 50.4 & 4.6 & 4.6 \\
$\triangle \mathrm{L} 006$ & Solid & 56.5 & 4.8 & 5.0 \\
$\triangle \mathrm{R} 001$ & Solid & 61.0 & 4.9 & 5.5 \\
$\triangle \mathrm{L} 007$ & Solid & 71.7 & 5.2 & 4.9 \\
$\triangle \mathrm{L} 001$ & Solid & 77.8 & 5.3 & 5.0 \\
$\triangle \mathrm{L} 008$ & Solid & 76.3 & 5.3 & 5.0 \\
$\triangle \mathrm{L} 003$ & Solid & 97.7 & 5.7 & 6.1 \\
$\triangle \mathrm{L} 004$ & Solid & 111.4 & 6.0 & 5.2 \\
$\triangle \mathrm{R} 002$ & Solid & 117.5 & 6.1 & 6.1 \\
$\triangle \mathrm{L} 002$ & Solid & 135.8 & 6.4 & 6.1 \\
$\triangle \mathrm{L} 009$ & Solid & 143.4 & 6.5 & 6.6 \\
$\triangle \mathrm{R} 003$ & Solid & 148.0 & 6.6 & 5.6
\end{tabular}

Fig. 1. Example of detection of a pulmonary nodule on a computed tomography simulation image. (A) Target nodule is indicated by the green circle. (B) List of marked nodules and their respective volume, diameter and pattern.

positive predictive value (PPV) were used.

Before calculations were performed, we defined the following terminologies: 1) true positive (TP) was defined as the significant nodule diagnosed as metastatic; 2) FP was defined as the significant nodule diagnosed as non-metastatic; 3 ) false negative (FN) was defined as the nodule considered insignificant diagnosed as metastatic; and 4) true negative (TN) was defined as the insignificant nodule eventually diagnosed as non-metastatic. Additionally, the patients who developed lung metastasis without initially detected lung nodule defined as FN. The patient with no detected nodule who eventually revealed to be non-metastatic defined as TN. Only CT images obtained within 4 months after RT completion were used for diagnosis of lung metastasis.

The sensitivity, NPV, and PPV were calculated for the number of nodules or the number of patients.

$$
\begin{gathered}
\text { Sensitivity }=T P /(T P+F N) \times 100 \\
N P V=T N /(T N+F N) \times 100 \\
P P V=T P /(T P+F P) \times 100
\end{gathered}
$$

\section{Results}

\section{Patient characteristics}

Patient characteristics are shown in Table 1. A total of 67 patients were analyzed. The median follow-up time for all patients was 10.1 months (range, 1 to 44.5 months). The median age of all patients was 55 years. Most of patients had vascular invasion (95.5\%) and multiple primary lesions (65.7\%). 
Table 1. Clinical characteristics of treatment naïve HCC patients $(n=67)$

\begin{tabular}{|c|c|}
\hline Characteristic & No. of patients (\%) \\
\hline \multicolumn{2}{|l|}{ Gender } \\
\hline Female & $10(14.9)$ \\
\hline Male & $57(85.1)$ \\
\hline Age (yr), median (range) & $55(27-76)$ \\
\hline \multicolumn{2}{|l|}{ ECIG performance } \\
\hline 0 & $23(34.3)$ \\
\hline 1 & $44(65.7)$ \\
\hline \multicolumn{2}{|l|}{ Liver cirrhosis } \\
\hline Yes & $41(61.0)$ \\
\hline No & $26(39.0)$ \\
\hline \multicolumn{2}{|l|}{ Child-Pugh score } \\
\hline$A$ & $58(86.6)$ \\
\hline B & $9(13.4)$ \\
\hline C & $0(0.0)$ \\
\hline \multicolumn{2}{|l|}{ AFP (IU) } \\
\hline$<400$ & $26(39.0)$ \\
\hline$\geq 400$ & $41(61.0)$ \\
\hline \multicolumn{2}{|l|}{ Tumor size $(\mathrm{cm})$} \\
\hline$<5$ & $15(22.0)$ \\
\hline$\geq 5$ & $52(78.0)$ \\
\hline \multicolumn{2}{|l|}{ Vascular invasion } \\
\hline No & $3(4.5)$ \\
\hline Yes & $64(95.5)$ \\
\hline \multicolumn{2}{|l|}{ Multiplicity } \\
\hline Single & $44(65.7)$ \\
\hline Multiple & $23(34.3)$ \\
\hline \multicolumn{2}{|l|}{ mUICC T stage } \\
\hline 1 & 0 \\
\hline 2 & $3(4.5)$ \\
\hline 3 & $22(32.8)$ \\
\hline 4 & $42(62.7)$ \\
\hline \multicolumn{2}{|l|}{ CLIP score } \\
\hline 0 & $23(34.3)$ \\
\hline 1 & $28(41.8)$ \\
\hline 2 & $16(23.9)$ \\
\hline
\end{tabular}

HCC, hepatocellular carcinoma; ECOG, Eastern Cooperative Oncology Group; AFP, alpha-fetoprotein; mUICC, modified Union for International Cancer Control; CLIP, Cancer of the Liver Italian Program.

\section{Nodules detected by computer-aided diagnosis alone on computed tomography simulation images}

Total number of CT simulation image was 67. Forty-six patients had incidental nodules detected by CAD on CT simulation images (Fig. 2). The total number of detected nodules was 109. These nodules were divided into two categories (Fig. 3). First, a 'significant nodule' was defined as a real lung nodule surrounded by lung parenchyma. A lung nodule is defined

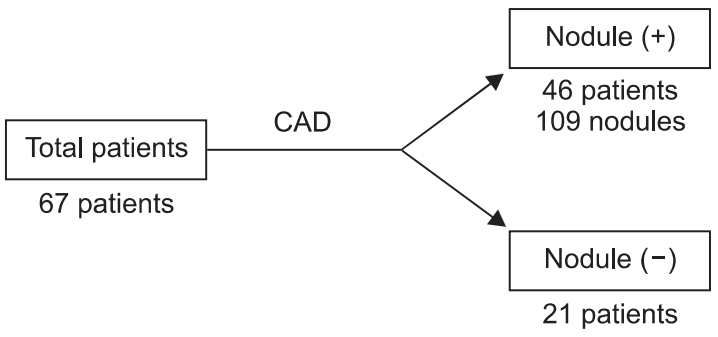

Fig. 2. The incidence of nodules detected by computer-aided diagnosis (CAD) alone $(n=67)$.

as a round opacity at least moderately well-marginated and no greater than $3 \mathrm{~cm}$ in maximum diameter [9]. Second, an 'insignificant nodule' was defined as a false nodule (e.g., intersection of bronchial or vascular structures, peribronchial thickening, or false nodular structure surrounded by bowel gas below the diaphragm).

The characteristics of the 109 nodules detected by CAD are summarized in Table 2. Most of the nodules were located in the lung (89.9\%), but 11 (10.1\%) nodules were located outside the lung. Only 20 (18.3\%) nodules were considered to be significant nodules. The remaining 89 (81.7\%) nodules were considered to be insignificant nodules.

\section{Nodules detected by integration of computer-aided diagnosis results and physician review on computed tomography simulation images}

Twenty significant nodules in 7 patients were detected by CAD alone (Fig. 4). Among those 20 significant nodules, the physician failed to detect 2 significant nodules in 2 patients. These 2 nodules were located in regions of increased haziness due to insufficient inflation of the lung. However, the physician detected 4 additional significant nodules in 2 patients that were not detected by CAD. The locations of nodules missed by automated detection by CAD were in the subpleural area, or adjacent to the diaphragm or pericardium. As a result, the total number of significant nodules detected by both CAD and the physician was 24 in 9 patients.

A total of 109 nodules were detected by CAD alone and 4 additional nodules were found after the review by a physician (Fig. 5A). Among the total of 113 nodules, 24 were found to be significant nodules while 89 nodules were categorized as insignificant nodules. Fig. 5B illustrates the corresponding number of patients with or without nodules after the review of images by CAD and a physician. Forty-six patients had nodules detected by CAD alone. The same number of patients was found to have nodules after review by both CAD and a 

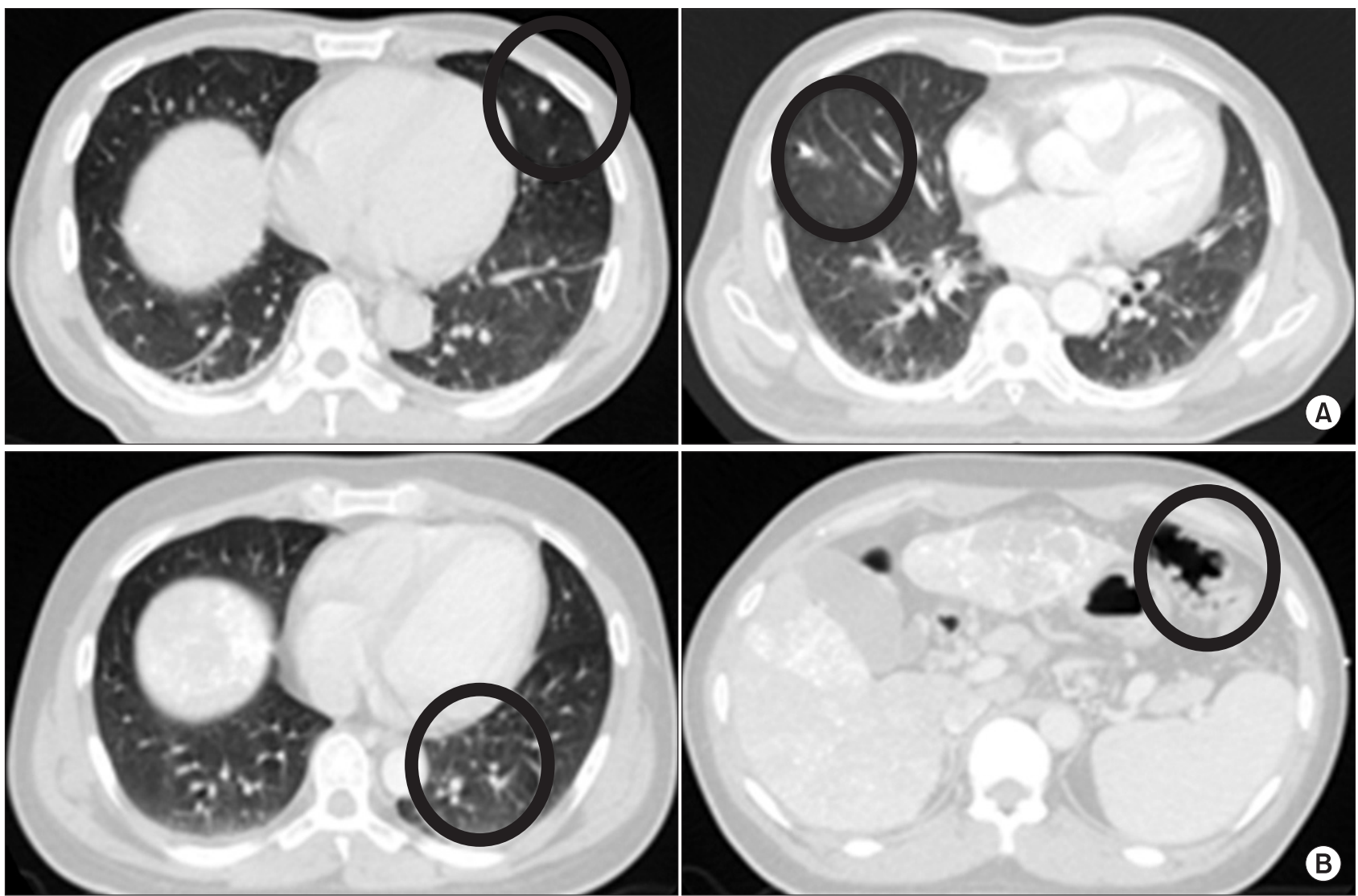

Fig. 3. The two categories of nodules detected by computer-aided diagnosis alone: (A) a significant nodule and (B) an insignificant nodule.

Table 2. Characteristics of lung nodules detected by CAD alone $(n=109)$

\begin{tabular}{lc}
\hline \multicolumn{1}{c}{ Characteristic } & No. of patients (\%) \\
\hline Location & \\
Lung & $98(89.9)$ \\
Other & $11(10.1)$ \\
Right/left & \\
Right & $50(45.9)$ \\
Left & $59(54.1)$ \\
Pattern & \\
Solid & $100(91.7)$ \\
GGO & $9(8.3)$ \\
Volume $\left(\mathrm{mm}^{3}\right)$, median (range) & $141.90(33.6-47,345.0)$ \\
Diameter $\left(\mathrm{mm}^{2}\right)$, median (range) & $6.50(4.0-44.9)$ \\
Categories of nodule & \\
$\quad$ Significant nodule & $20(18.3)$ \\
Insignificant nodule & $89(81.7)$ \\
\hline
\end{tabular}

$C A D$, computer-aided diagnosis; GGO, ground glass opacity.

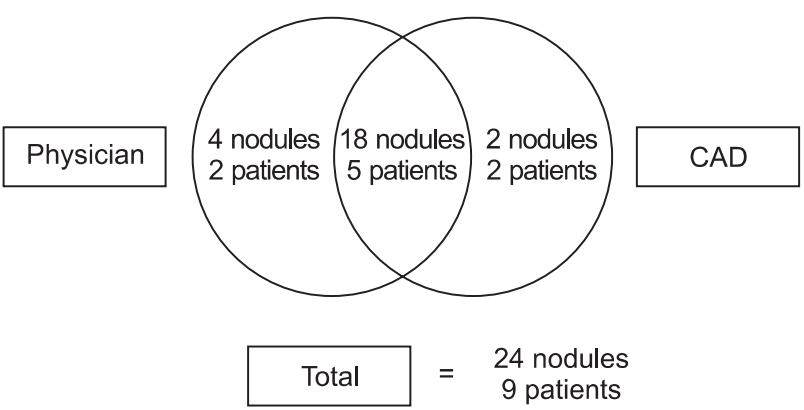

Fig. 4. A schematic diagram of the number of significant nodules and the number of patients with significant nodules detected by integration of the computer-aided diagnosis (CAD) result and the physician review.

physician. Among those patients, 9 patients had the significant nodules and 37 patients had insignificant nodules.

The differences between the patients with significant lung nodules and the remainder of the patients are summarized in Table 3. There were no significant differences between the two groups. 
A

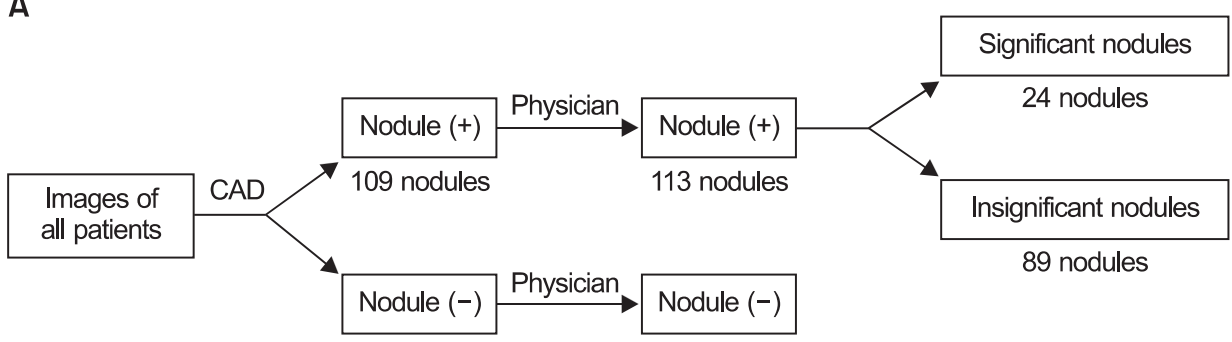

B

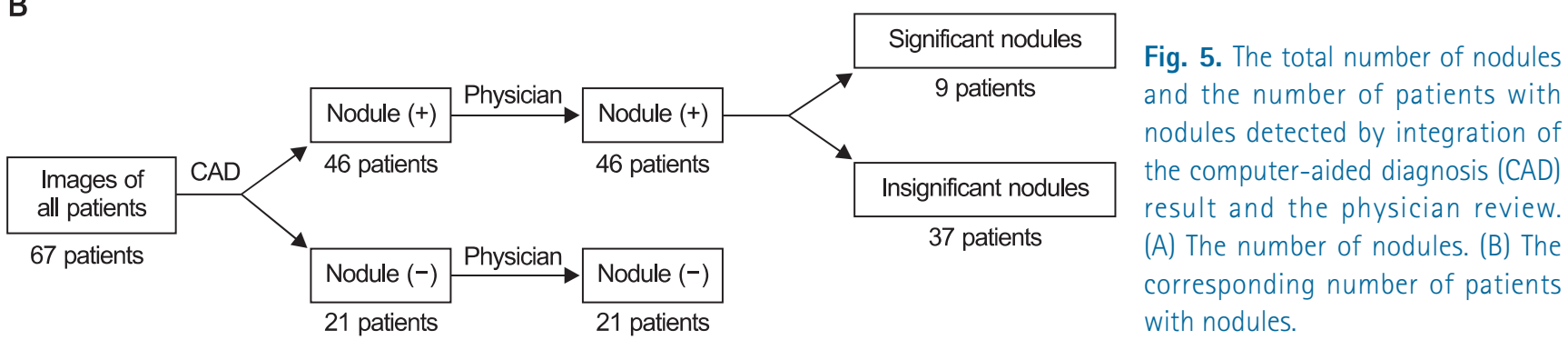

\section{The fate of the incidental lung nodules on computed tomography simulation images}

During follow-up, lung metastasis was found in 12 patients. The median time to synchronous lung metastases was 1.1 months (range, 0.9 to 3.8 months). The correlation between the development of a lung metastasis and the review results of the CT simulation images by CAD and a physician is presented in Fig. 6. Lung metastasis developed in 11 of 46 patients with any type of nodule (Fig. 6A). Among 9 patients with significant nodules, 7 patients developed a lung metastasis. In patients without significant nodules, 4 patients developed a lung metastasis. Only one patient among those patients without nodules developed a lung metastasis.

The number of nodules diagnosed as metastatic lung nodules was 22 among the 113 nodules detected after the review of CT simulation images (Fig. 6B). Among the 24 significant nodules, 22 nodules were eventually diagnosed as metastatic lung nodules. None of the 89 insignificant nodules became a metastatic nodule.

The corresponding bins of TP, FP, TN, and FN are diagrammed in Fig. 6. The sensitivity was 58.3\% calculated based on patient number. When calculated based on the number of nodules, sensitivity was 100\%. The NPVs were $91.4 \%$ and $100 \%$ based on patient number and on the number of nodules, respectively. PPVs of $77.8 \%$ and $91.7 \%$ were calculated based on patient number and number of nodules, respectively.

\section{Discussion and Conclusion}

HCC is a leading cause of cancer death with a high incidence in Asian countries. Although prognosis has improved as a result of advances in diagnostic techniques and treatment modalities, it still remains poor, with a mean survival of 6-20 months [10]. Hepatic resection offers the best chance for a possible cure, but less than $20 \%$ of patients are eligible for resection at the time of diagnosis. Treatment options for unresectable HCC are TACE, RT alone or combination therapy including TACE or sorafenib [11-14]. For advanced HCC with distant metastases, coexisting extrahepatic lesions are usually not treated by loco-regional therapies. In randomized controlled trials, the multikinase inhibitor sorafenib resulted in prolonged survival and is consequently considered a standard treatment for such patients [15].

The most frequent site of extrahepatic metastasis is the lung and the median survival for patients with lung metastasis is about 8 months [16]. Due to the high incidence of extrahepatic metastases which occur in up to $40 \%$ of HCC cases, chest imaging is recommended as a part of the initial workup. CT is currently the best imaging modality for evaluating lung nodules because of its spatial and contrast resolution. If chest X-ray is the only available chest imaging method, CT simulation for RT could be the first imaging modality used for more precise lung evaluation, despite the fact that the whole lung is not scanned. The incidental finding of metastatic nodules was not an uncommon event. Twenty-two of 113 (19.5\%) nodules and 22 of 24 (91.7\%) significant nodules 
Table 3. Comparison of the clinical characteristics of patients with significant nodules with the rest of the patients $(n=67)$

\begin{tabular}{|c|c|c|c|}
\hline Characteristic & $\begin{array}{c}\text { Patients with significant } \\
\text { nodules }(n=9)\end{array}$ & $\begin{array}{c}\text { Patients without significant } \\
\text { nodules }(n=58)\end{array}$ & $\mathrm{p}$-value \\
\hline Sex & & & 0.990 \\
\hline Female & 0 & $10(17.2)$ & \\
\hline Male & $9(100)$ & $48(82.8)$ & \\
\hline Age (yr) & $54(49-68)$ & $56(27-76)$ & 1.020 \\
\hline ECOG performance & & & 0.946 \\
\hline 0 & $3(33.3)$ & $20(34.5)$ & \\
\hline 1 & $6(66.7)$ & 38 (65.5) & \\
\hline Child-Pugh score & & & 0.343 \\
\hline A & $9(100)$ & $49(84.5)$ & \\
\hline B & 0 & $9(15.5)$ & \\
\hline C & 0 & 0 & \\
\hline Vascular invasion & & & 1.000 \\
\hline No & 0 & $3(5.2)$ & \\
\hline Yes & $9(100)$ & $55(94.8)$ & \\
\hline Multiplicity & & & 0.495 \\
\hline Multiple & $5(55.5)$ & $39(67.2)$ & \\
\hline Single & $4(44.5)$ & 19 (32.8) & \\
\hline mUICC T stage & & & 0.793 \\
\hline 1 & 0 & 0 & \\
\hline 2 & 0 & $3(5.2)$ & \\
\hline 3 & $4(44.5)$ & $18(31.0)$ & \\
\hline 4 & $5(55.5)$ & $37(63.8)$ & \\
\hline CLIP score & & & 0.826 \\
\hline 0 & $3(33.3)$ & $20(34.5)$ & \\
\hline 1 & $4(44.5)$ & $24(41.4)$ & \\
\hline 2 & $2(22.2)$ & $14(24.1)$ & \\
\hline Lung parenchymal change after TACE & & & 0.923 \\
\hline No & $6(69.7)$ & $26(44.8)$ & \\
\hline Yes & $3(33.3)$ & $32(55.2)$ & \\
\hline
\end{tabular}

Values are presented as number (\%) or median (range).

ECOG, Eastern Cooperative Oncology Group; mUICC, modified Union for International Cancer Control; CLIP, Cancer of the Liver Italian Program.

(detected by CAD and a physician) were metastatic nodules in our study. To avoid unnecessary treatment, a radiation oncologist should review the CT simulation images carefully to detect unexpected incidental findings, such as a lung metastasis. Also, re-evaluation of treatment modalities is needed if a significant nodule is found on image review due to the high likelihood of lung metastases.

Some studies have recommended routine interpretation of simulation images by diagnostic radiologists [3]. This recommendation can be difficult to follow depending on the clinical situation. Also, lung nodules present on a chest $\mathrm{CT}_{\text {, }}$ especially those smaller than $5 \mathrm{~mm}$, can be easily missed by the physician when using only a visual assessment. Numerous studies have shown that CAD has allowed a significant increase in diagnostic accuracy and an improvement in the performance of physician in detecting lung nodules [6]. These benefits are particularly evident with inexperienced physicians [17]. In a large observer study by Brown et al. [18], the use of a CAD system for nodule detection resulted in a 12\%-23\% increase in detection rate and equalized the detection rate between radiologists and non-radiologists. Similarly, in our study, use of CAD resulted in the detection of 2 additional significant nodules which were undetected by the physician during visual inspection.

The computer output is generally used by physicians as a second opinion in order to improve upon the sensitivity of a human reader. In the study by Beyer et al. [19], the reading time was 274 seconds when CAD was used to preselect lung 
A

\begin{tabular}{|c|c|c|c|c|c|}
\hline & \multirow[b]{3}{*}{$\begin{array}{l}\text { Integrated review } \\
\text { (CAD + physician) }\end{array}$} & \multirow[b]{2}{*}{ Nodule (+) } & & $\begin{array}{c}\text { With significant } \\
\text { nodules }\end{array}$ & $\begin{array}{c}\text { Without significant } \\
\text { nodules }\end{array}$ \\
\hline & & & $\begin{array}{l}\text { Lung } \\
\text { metastasis }\end{array}$ & $\begin{array}{l}7 \text { patients } \\
\text { TP }\end{array}$ & $\begin{array}{l}4 \text { patients } \\
\text { FN }\end{array}$ \\
\hline \multirow{2}{*}{$\begin{array}{l}\text { Images of } \\
\text { all patients }\end{array}$} & & 46 patients & $\begin{array}{c}\text { No } \\
\text { metastasis }\end{array}$ & $\begin{array}{l}2 \text { patients } \\
\text { FP }\end{array}$ & $\begin{array}{l}33 \text { patients } \\
\text { TN }\end{array}$ \\
\hline & & & & & \\
\hline \multirow[t]{2}{*}{67 patients } & & Nodule (-) & $\begin{array}{l}\text { Lung } \\
\text { metastasis }\end{array}$ & $\begin{array}{l}1 \text { patients } \\
\text { FN }\end{array}$ & \\
\hline & & 21 patients & $\begin{array}{c}\text { No } \\
\text { metastasis }\end{array}$ & $\begin{array}{l}20 \text { patients } \\
\text { TN }\end{array}$ & \\
\hline
\end{tabular}

B

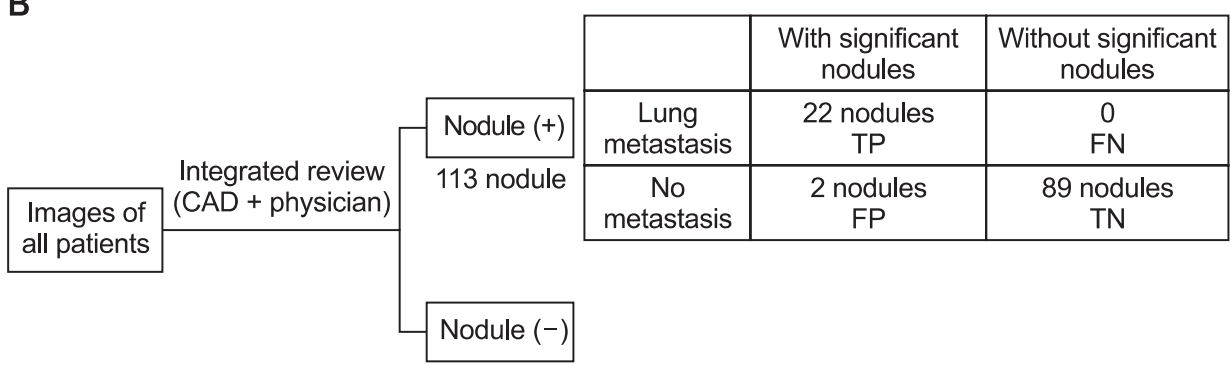

Fig. 6. The correlation between the development of lung metastasis and the results of computed tomography simulation review by computer-aided diagnosis (CAD) and a physician. (A) The number of patients. (B) The number of nodules. TP, true positive; FN, false negative; FP, false positive; $\mathrm{TN}_{\mathrm{N}}$ true negative. nodules prior to reading by a physician. Although the reading time was longer when CAD was used as a second reader to check out overlooked nodules after reading by a physician, the sensitivity was significantly higher. Due to the multiplicative benefits of utilizing CAD as a concurrent reader, use of CAD has become widespread in clinical situations. However, CAD had high FP rate. Furthermore, nodules not surrounded by lung parenchyma are likely to be missed, especially in subpleural, fissural, and costophrenic angle areas [20,21]. Moreover, CAD analysis is a complex procedure.

There are some considerations in interpreting our results. First, the slice thickness of the CT simulation image was thicker than those used in previous studies. A correlation between section thickness and sensitivity of CAD has been previously reported by Kim et al. [22]. The sensitivity was 95.2\% for the 1-mm slice thickness group and 88.5\% for the $5-\mathrm{mm}$ slice thickness group, respectively. Second, our CT simulation images did not cover the whole lung and included the abdominal cavity with loculation of bowel gas in the scanning range. Various segmented vascular structures might lead to misinterpretation of CAD [21]. Also images of other organs might contribute to the high number of insignificant nodules detected. Third, the detection rate by physicians in our study may have been higher than those of a typical clinical practice. Study physicians may have reviewed the images more thoroughly than usual and may therefore have influenced the detection rate in the current study.
In order for wide usage of CAD systems to become standard practice, the diagnostic accuracy will need to be improved. Furthermore, in order to improve accessibility for radiation oncologists, the development of a CAD system which is combined with an RT planning system would be required.

\section{Conflict of Interest}

No potential conflict of interest relevant to this article was reported.

\section{Acknowledgments}

This research was supported by Basic Science Research Program through the National Research Foundation of Korea (NRF) funded by the Ministry of Education, Science and Technology (NRF-2012R1A1A2042414), and Samsung Medical Center grant (GF01130081).

\section{References}

1. Kim MJ, Kim EK, Keum KC, Park BW, Son EJ, Oh KK. Treatmentplanning CT scan for breast and chest-wall irradiation: how many unexpected abnormalities could we detect? Clin Imaging 2008;32:443-6.

2. Mehta VK, Goffinet DR. Unsuspected abnormalities noted on CT treatment-planning scans obtained for breast and chest 
wall irradiation. Int J Radiat Oncol Biol Phys 2001;49:723-5.

3. Nakamura N, Tsunoda $H$, Takahashi 0 , et al. Frequency and clinical significance of previously undetected incidental findings detected on computed tomography simulation scans for breast cancer patients. Int J Radiat Oncol Biol Phys 2012;84:602-5.

4. Smitt MC, Mehta VK. Is diagnostic review of radiotherapyplanning CT scans important in the conformal therapy era? AJR Am J Roentgenol 2001;177:521-4.

5. Doi K. Computer-aided diagnosis in medical imaging: historical review, current status and future potential. Comput Med Imaging Graph 2007;31:198-211.

6. Fraioli F, Serra G, Passariello R. CAD (computed-aided detection) and CADx (computer aided diagnosis) systems in identifying and characterising lung nodules on chest CT: overview of research, developments and new prospects. Radiol Med 2010;115:385-402.

7. Seo JB, Im JG, Goo JM, Chung MJ, Kim MY. Atypical pulmonary metastases: spectrum of radiologic findings. Radiographics 2001;21:403-17.

8. Hirakata $K$, Nakata $H$, Nakagawa T. CT of pulmonary metastases with pathological correlation. Semin Ultrasound CT MR 1995;16:379-94.

9. Austin JH, Muller NL, Friedman PJ, et al. Glossary of terms for CT of the lungs: recommendations of the Nomenclature Committee of the Fleischner Society. Radiology 1996;200:32731.

10. Bialecki ES, Di Bisceglie AM. Diagnosis of hepatocellular carcinoma. HPB (Oxford) 2005;7:26-34.

11. Choi JY. Treatment algorithm for intermediate and advanced stage hepatocellular carcinoma: Korea. Oncology 2011;81 Suppl 1:141-7.

12. Kim Yl, Park HC, Lim DH, et al. Changes of the liver volume and the Child-Pugh score after high dose hypofractionated radiotherapy in patients with small hepatocellular carcinoma.
Radiat Oncol J 2012;30:189-96.

13. Park HC, Seong J, Tanaka M, et al. Multidisciplinary management of nonresectable hepatocellular carcinoma. Oncology 2011;81 Suppl 1:134-40.

14. Yu Jl, Park HC, Lim DH, et al. Scheduled interval trans-catheter arterial chemoembolization followed by radiation therapy in patients with unresectable hepatocellular carcinoma. J Korean Med Sci 2012;27:736-43.

15. Llovet JM, Ricci S, Mazzaferro V, et al. Sorafenib in advanced hepatocellular carcinoma. N Engl J Med 2008;359:378-90.

16. Uchino $K$, Tateishi $R$, Shiina $S$, et al. Hepatocellular carcinoma with extrahepatic metastasis: clinical features and prognostic factors. Cancer 2011;117:4475-83.

17. Awai $K$, Murao K, Ozawa A, et al. Pulmonary nodules: estimation of malignancy at thin-section helical CT: effect of computer-aided diagnosis on performance of radiologists. Radiology 2006;239:276-84.

18. Brown MS, Goldin JG, Rogers S, et al. Computer-aided lung nodule detection in $\mathrm{CT}$ : results of large-scale observer test. Acad Radiol 2005;12:681-6.

19. Beyer F, Zierott L, Fallenberg EM, et al. Comparison of sensitivity and reading time for the use of computer-aided detection (CAD) of pulmonary nodules at MDCT as concurrent or second reader. Eur Radiol 2007;17:2941-7.

20. Song KD, Chung MJ, Kim HC, Jeong SY, Lee KS. Usefulness of the CAD system for detecting pulmonary nodule in real clinical practice. Korean J Radiol 2011;12:163-8.

21. Goo JM. A computer-aided diagnosis for evaluating lung nodules on chest $\mathrm{CT}$ : the current status and perspective. Korean J Radiol 2011;12:145-55.

22. Kim JS, Kim JH, Cho G, Bae KT. Automated detection of pulmonary nodules on CT images: effect of section thickness and reconstruction interval: initial results. Radiology 2005; 236:295-9. 